\title{
СОВРЕМЕННОЕ ПРЕДСТАВЛЕНИЕ О ХРОНИЧЕСКОЙ СЕРДЕЧНОЙ НЕДОСТАТОЧНОСТИ
}

ЭЛЬВИРА БАКИЕВНА ФРОЛОВА, зам. начальника по лечебной работе ФКУЗ «Медико-санитарная часть МВД РФ по Республике Татарстан», Казань, Россия, e-mail: frolova.67@mail.ru

МАРАТ ФАРИДОВИЧ ЯУШЕВ, докт. меД. наук, проф. кафедры фтизиопульмонологии ГБОУ ВПО «Казанский

государственный медицинский университет» Минздрава России, e-mail: umukgmu@rambler.ru

Реферат. В настоящее время XCH рассматривается как симптомокомплекс с абсолютно неблагоприятным прогнозом. Несмотря на все достижения современной медицины, ХCH ложится тяжелым бременем не только на российское, но и на мировое здравоохранение. Большое число научных исследований, проведенных за последнее время, позволяет глубже понять его патогенез. По данным эпидемиологических исследований последних 10 лет, проведенных в нашей стране, в рамках исследований ЭПОХА-ХСН и ЭПОХА-О-ХСН стало известно, что в РФ распространенность в популяции XCH I-IV ФК составила 7\% случаев (7,9 млн человек), клинически выраженная XCH (II-IV ФК) имеет место у 4,5\% населения (5,1 млн человек), распространенность терминальной XCH (III-IV ФК) достигает 2,1\% случаев (2,4 млн человек). Основную долю составляли больные ХCH в возрасте старше 60 лет, а распространенность этого состояния была достоверно выше среди женщин. По данным Фрамингемского исследования, показатели смертности среди больных ХCH уменьшаются в среднем на 12\% в декаду, тем не менее за период с 1990 по 1999 г. средняя 5-летняя смертность в популяции больных XCH (I-IV ФК) остается высокой и составляет 59\% для мужчин и 45\% для женщин, а встречаемость XCH в популяции имеет нарастающую динамику. Этиологическими факторами чаще всего являлись артериальная гипертония, ИБС. Тяжесть симптомов ХСН являлась достоверным предиктором плохого прогноза. Диагностика хронической сердечной недостаточности включает клиническое обследование и использование современных методов исследования.

Ключевые слова: хроническая сердечная недостаточность, диагностика, лечение.

\section{CURRENT UNDERSTANDING OF CHRONIC HEART FAILURE}

\author{
ELVIRA B. FROLOVA, MARAT F. YAUSHEV
}

\begin{abstract}
Chronic heart failure is usual cardiologic disease. Many scientific investigations made in recent time permeated to understand its causes. Investigations in Russian Federation showed $12,3 \%$ of population have heart failure and $2,3 \%$ have NYHA class III-IV. Most patients older 60 years and women. Heart failure was result of hypertension, ischemic disease and diabetes. In Framingham study lethality of heart failure decrease on $12 \%$ in decade, but lethality in chronic heart failure population NYHA class I-IV is high $59 \%$ for men and $45 \%$ for women and increase. Diagnosis of heart failure involves clinical investigation and current diagnostic methods. Treatment of chronic heart failure use many groups of medicines and their number is increasing. In Russian Federation chronic heart failure diagnosed like a syndrome, but in European countries and USA like disease.
\end{abstract}

Key words: chronic heart failure, diagnostic, treatment.

$\mathrm{B}$ настоящее время хроническая сердечная недостаточность $(\mathrm{XCH})$ рассматривается как симптомокомплекс с абсолютно неблагоприятным прогнозом. Несмотря на все достижения современной медицины, $\mathrm{XCH}$ ложится тяжелым бременем не только на российское, но и на мировое здравоохранение [1].

Впервые хроническая сердечная недостаточность (XCH) заявила о себе как серьезная общественная проблема в 1960 г., когда госпитальная статистика США зарегистрировала своеобразный рекорд: число больных ХСН превысило $1 \%$ от всех госпитализированных в стационары, а частота впервые установленного диагноза ХCH составила 2 на 1000 всех обращений в год [2]. При этом общее по стране число больных ХСН составило 1,4 млн человек. Согласно расчетам T. Gibson и соавт. (1966), число пациентов, страдающих ХCH, в 80-е гг. должно было увеличиться до 1,7-1,9 млн человек [3]. Однако реальная картина 80-х гг. превзошла все ожидания: в 1989 г. число госпитализаций по поводу $\mathrm{XCH}$ увеличилось в 2 раза (2\%), а число вновь выявленных случаев заболевания возросло до 2,5-2,7 на 1000 [4]. Дополнительно к этому до 4\% всех госпитализированных имели ХCH в качестве сопутствующего заболевания. В целом сердечной недостаточностью в конце 80-х гг. страдало до 4 млн американцев (вместо 1,9 млн расчетных), что составляло примерно 1,5\% от численности взрослого населения страны, и их число увеличивалось на 400 тыс. ежегодно [5].

Отдельные сообщения свидетельствуют об аналогичной США картине распространения заболевания и в нашей стране [6].

По данным эпидемиологических исследований последних 10 лет, проведенных в нашей стране, в рамках исследований ЭПОХА-ХСН и ЭПОХА-О-ХСН стало известно, что в РФ распространенность в популяции XCH I-IV ФК составила 7\% случаев (7,9 млн человек), 
клинически выраженная XCH (II-IV ФК) имеет место у $4,5 \%$ населения (5,1 млн человек), распространенность терминальной XCH (III-IV ФК) достигает 2,1\% случаев (2,4 млн человек). Среди мужчин распространенность $\mathrm{XCH}$ выше, чем среди женщин, в возрастных группах до 60 лет, что ассоциируется с более ранней заболеваемостью АГ и ИБС у мужчин [7]. За счет большей продолжительности жизни число женщин, имеющих $\mathrm{XCH}$, в 2,6 раза превосходит число мужчин (72\% против 28\%) [8].

В исследовании Euro Heart Survey, проводившемся в 14 странах Европы, включая РФ, впервые особое внимание было уделено появлению большого числа пациентов с ХСН и нормальной (ФВ > 50 \%) систолической функцией сердца [9]. По данным исследования ЭПОХА-О-ХСН, в РФ 56,8\% пациентов с очевидной ХСH имеют практически нормальную сократимость миокарда (ФВ ЛЖ > 50\%) [10].

Заболеваемость ХСН растет с возрастом: этот показатель у европейских мужчин 70-79 лет составляет 13/1000, у женщин - 9/1000 [11]. В возрасте 80-89 лет заболеваемость $\mathrm{XCH}$ увеличивается до 27/1000 у мужчин и 22/1000 у женщин [12-14]. ХСН является наиболее частым диагнозом при выписке из стационаров у пациентов старше 65 лет, этот синдром также является наиболее частой причиной повторных госпитализаций [15]. Количество госпитализаций с диагнозом ХCH в период с 1979 по 1999 г. возросло на 155\%, смертность от ХCH в эти же сроки выросла на 145\% [16].

Годовая смертность от ХСН в РФ достоверно выше, чем в популяции (отношение шансов 10,3). Среди пациентов с XCH I-IV ФК средняя годовая смертность составляет 6\% [17]. При этом однолетняя смертность больных с клинически выраженной ХCH достигает $12 \%$, даже в условиях лечения в специализированном стационаре, т.е. за один год в РФ умирают до 612 тыс. больных ХCH [18].

Таким образом, по масштабам и скорости распространения XCH сопоставима с самыми опасными инфекционными эпидемическими заболеваниями [19].

Несмотря на очевидные успехи последних десятилетий в области изучения патогенеза и поисков эффективных путей лечения, ХCH по-прежнему остается одним из самых тяжелых и прогностически неблагоприятных заболеваний сердечно-сосудистой системы [20, 21, 22].

По данным Фрамингемского исследования, показатели смертности среди больных ХCH уменьшаются в среднем на $12 \%$ в декаду, тем не менее за период с 1990 по 1999 г. средняя 5-летняя смертность во всей популяции больных XCH (I-IV ФК) остается высокой и составляет 59\% для мужчин и $45 \%$ для женщин, а встречаемость ХCH в популяции имеет нарастающую динамику [23].

Вклад различных нозологий в структуру заболеваемости ХCH значительно отличается в зависимости от типа исследования. В популяционных работах самой частой причиной ХCH является артериальная гипертония (АГ). Так, например, по данным Фрамингемского исследования, АГ в «чистом» виде или в комбинации с ИБС составляет 70\% всех причин ХCH у мужчин и 78\% у женщин [23]. В то же время при исследовании пациентов, находящихся на стационарном лечении, основной причиной декомпенсации является ИБС и некоронарогенные поражения миокарда [19].
Основными этиологическими причинами ХСН в России являются АГ (88\% случаев) и ИБС $(59 \%$ случаев) [18]. В РФ можно отметить еще три важные причины развития $\mathrm{XCH}$ : хроническую обструктивную болезнь легких (ХОБЛ) - 13\% случаев, сахарный диабет (СД) - 11,9\% случаев и перенесенное острое нарушение мозгового кровообращения (ОНМК) - 10,3\% случаев. Наличие большого количества ФР приводит к более раннему развитию причин $\mathrm{XCH}$, что становится базисом для более раннего формирования $\mathrm{CH}$ в возрастных группах до 60 лет с достоверно более плохим прогнозом жизни больных в последующие десятилетия [24-25].

\section{Современные представления о патогенезе XCH}

При рассмотрении патогенеза сердечной недостаточности следует отметить, что ХСН - это синдром, развивающийся в результате различных заболеваний сердечно-сосудистой системы, приводящих к снижению насосной функции сердца (хотя и не всегда), дисбалансу между гемодинамической потребностью организма и возможностями сердца, хронической гиперактивации нейрогормональных систем, и проявляющийся одышкой, сердцебиением, повышенной утомляемостью, ограничением физической активности и избыточной задержкой жидкости в организме.

Принципиальная новизна современных представлений о патогенезе ХCH связана с тем фактом, что далеко не все больные имеют симптомы декомпенсации в результате снижения насосной способности сердца. Тремя ключевыми событиями на пути развития и прогрессирования XСН являются: заболевание сердечнососудистой системы; снижение сердечного выброса (у большинства больных); задержка натрия и избыточной жидкости в организме.

На самом деле в организме имеется система сдержек и противовесов и все основные нейрогормональные системы можно разделить условно на вызывающие вазоконстрикцию, пролиферацию (ремоделирование органов) и антидиурез и противодействующие им вазодилатирующие, диуретические и антипролиферативные (тормозящие ремоделирование) системы. С одной стороны, находятся симпатико-адреналовая система (САС) и ее эфффекторы НА, адреналин и РАAC и ее эффректоры ангиотензин II (A II), а также эндотелин, вазопрессин. С другой стороны, им противостоят система натрийуретических пептидов, брадикинин (БК), вазодилатирующие простаноиды, оксид азота (NO) и некоторые другие [26].

Вместе с тем общеизвестно, что нейрогуморальная дисрегуляция, занимая одну из ключевых позиций в развитии $\mathrm{XCH}$, отнюдь не является единственным и тем более пусковым механизмом ее патогенеза. Очевидно, что развитие $\mathrm{XCH}$ невозможно без снижения насосной способности сердца, которая определяется эффрективностью его систолической и диастолической функций [27].

По определению ВОЗ (1995), сердечная недостаточность с патофизиологической точки зрения представляет собой неспособность сердца обеспечивать питательными веществами ткани организма в соответствии с их метаболическими потребностями в состоянии покоя и/или при умеренных физических нагрузках. Сердечная недостаточность является многосистемным заболеванием, которое вовлекает сердце, 
периферическое сосуды, почки, симпатическую нервную систему, ренин-ангиотензиновую систему, другие циркулирующие гормоны, локальные паракринную и аутокринную системы и метаболические процессы в скелетной мускулатуре.

Нарушение насосной функции сердца лежит в основе сердечной недостаточности: сердечная мышца не способна в большей или меньшей степени выталкивать кровь в сосудистое русло в систолу и/или адекватно наполняться в диастолу. При этом клинически наблюдаются снижение переносимости физической нагрузки (утомляемость), одышка, задержка жидкости в организме (приводит к возникновению периферических отеков и отеку легких). Неуклонное прогрессирующее течение заболевания связано с ухудшением структуры и функции миокарда, что в результате существенно сокращает продолжительность жизни больных [28].

V. Dzau и E. Braunwald в 1991 г. сфрормулировали понятие единого «сердечно-сосудистого континуума», которое в течение последних 20 лет конца XX и начала XXI в. прочно вошло в научно-медицинскую практику Применительно к практической медицине «континуум» (от английского «continuous» - постоянный, непрерывный) подразумевает непрерывную последовательность этапов развития заболевания - от ФР до летального исхода [29].

\section{Сердечно-сосудистый континуум}

В 2001 г. на совместном заседании Американского колледжа и Американской ассоциации сердца были разработаны и рекомендованы этапы (стадии) сердечно-сосудистого континуума:

стадия А - это формирование ФР, начало профилактики и лечения каких-либо первых признаков заболевания с целью предупреждения развития $\mathrm{XCH}$;

стадия В - это годы лечения с использованием всех лечебно-профилактических мероприятий, включением методов эффективного восстановления насосной деятельности сердца;

стадия C - это месяцы лечения с помощью всех мероприятий стадий А и В с использованием лекарственных средств, направленных на устранение осложнений и клинических признаков $\mathrm{CH}$;

стадия D - самая сложная стадия, когда пациент нередко нуждается в наблюдении и лечении в блоках интенсивной терапии, в ряде случаев врачи вынуждены применять кардиореанимационные мероприятия подсадку искусственного левого желудочка, клеточную терапию, пересадку сердца, т.е. то, что требует серьезных фринансовых затрат [30-31].

Прогноз больных ХCH по-прежнему остается одним из самых плохих. XCH существенным образом влияет на летальность (L. Cupplles et al., 1988) [32]. Уже в 1971 г. при очередном анализе результатов Фрамингемского наблюдения стало очевидно, что прогноз $\mathrm{XCH}$ сопоставим с таковым у онкологических больных (McKeep A. et al., 1971).

Важнейшая роль в развитии и прогрессировании сердечной недостаточности принадлежит систолической функции левого желудочка. Трудно переоценить значение систолической функции для оценки прогноза больных XCH: в большинстве исследований величина фрракции выброса и другие индексы сократимости показали себя независимыми предикторами смертности и выживаемости больных XСН как при моно-, так и при многофакторном анализе (Мазур Н.А., 1996; Madsen B. et al., 1994; A. Gradman et al., 1989) [32].

Можно признать, что систолическая функция вполне оправдывает роль «сердечного» маркера заболевания и предиктора выживаемости больных ХСН. Увеличение объема полости левого желудочка и снижение фракции выброса сопряжены с повышенным риском развития $\mathrm{XCH}$ и смерти, увеличение сердца по результатам рентгенологических исследований ассоциируется с троекратным увеличением смертности среди больных, перенесших инфаркт миокарда (Davies S. et al., 1992) [32].

Хорошо известно, что падение сердечного выброса при снижении сократительной функции миокарда компенсируется двумя механизмами: механизмом Франка-Старлинга и активацией нейрогуморальных систем. Активация симпатоадреналовой системы (САC), ренин-ангиотензин-альдостероновой системы (PAAC) и системы аргинин-вазопрессина (АВП) приводит к повышению тонуса сосудов, задержки натрия и воды с увеличением объема циркулирующей крови. Кроме того, эти процессы запускают механизм длительной компенсации, главным компонентом которого является ремоделирование.

Процесс ремоделирования сердца при ХCH может проявляться по-разному. У части больных он носит адаптивный характер и приводит к уменьшению размеров полости левого желудочка, сохранению геометрически более выгодной цилиндрической формы левого желудочка, поддержанию сократительной способности его и стабилизации миокардиального стресса. У другой части больных ремоделирование приводит к прогрессирующей дилатации полости левого желудочка с переходом к гемодинамически более невыгодной сферической форме желудочка, росту миокардиального стресса и постепенному снижению сократительной фрункции желудочка. Эти морфоорункциональные изменения носят дезадаптивный характер и приводят к появлению клинических признаков ХCH (Никитин Н.П. и др., 1999).

Таким образом, процесс ремоделирования сердца у различных больных неоднозначен и может носить как адаптивный, так и дезадаптивный характер, что зависит, по-видимому, от сложного взаимодействия определяющих его факторов, в том числе генетического [32].

\section{Стадии и функциональные классы хронической сердечной недостаточности}

Различают острую и хроническую сердечную недостаточность $(\mathrm{CH})$. Под острой СН принято подразумевать возникновение острой (кардиогенной) одышки, связанной с быстрым развитием легочного застоя вплоть до отека легких или кардиогенного шока (с гипотонией, олигурией и т.д.), которые, как правило, являются следствием острого повреждения миокарда, прежде всего острого ИМ. Чаще встречается хроническая форма $\mathrm{CH}$, для которой характерны периодически возникающие эпизоды обострения (декомпенсации), проявляющиеся внезапным или, что бывает чаще, постепенным усилением симптомов и признаков ХСН. Постановка диагноза ХCH возможна при наличии двух ключевых критериев: характерных симптомов СН (главным образом одышки, утомляемости и ограничении физической активности, отеков лодыжек); объективного доказательства того, 
что эти симптомы связаны с повреждением сердца, а не каких-либо других органов [33].

По поводу классификации хронической сердечной недостаточности ведется немало споров в кардиологической среде. У нас в стране долгое время использовалась и используется до сих пор классификация В.Х. Василенко и Н.Д. Стражеско, предложенная ими на XII съезде терапевтов в 1935 г., т.е. более полувека назад:

Стадия I - начальная, скрытая недостаточность кровообращения, проявляющаяся только при физической нагрузке (одышка, сердцебиение, чрезмерная утомляемость). В покое эти явления исчезают. Гемодинамика не нарушена.

Стадия II - выраженная длительная недостаточность кровообращения, нарушения гемодинамики (застой в малом и большом кругах кровообращения) выражены в покое.

Период А - признаки недостаточности кровообращения в покое выражены умеренно. Нарушения гемодинамики лишь в одном из отделов сердечнососудистой системы (в большом или малом круге кровообращения).

Период Б - окончание длительной стадии, выраженные гемодинамические нарушения, в которые вовлечена вся сердечно-сосудистая система (и большой, и малый круг кровообращения).

Стадия III — конечная, дистрофическая с тяжелыми нарушениями гемодинамики, стойкими изменениями обмена веществ и необратимыми изменениями в структуре органов и тканей.

В настоящее время в нашей стране все чаще используется классификация сердечной недостаточности, предложенная Нью-Йоркской ассоциацией кардиологов (NYHA) еще в 1928 г. и пересмотренная в 1994 г. Согласно этой классификации больные с синдромом сердечной недостаточности разделены на четыре функциональных класса (ФК):

Класс І. Нет ограничений фризической активности и влияния на качество жизни пациента.

Класс II. Слабые ограничения фризической активности и полное отсутствие неудобств во время отдыха.

Класс III. Ощутимое снижение работоспособности, симптомы исчезают во время отдыха.

Класс IV. Полная или частичная потеря работоспособности, симптомы сердечной недостаточности и боль в груди проявляются даже во время отдыха.

Предложен также легкий и удобный способ определения ФК каждого пациента - так называемый «шестиминутный тест ходьбы». Для проведения теста достаточно попросить пациента в течение шести минут походить в удобном для него темпе и замерить потраченное на это время. Этого достаточно для расчета максимального потребления кислорода при нагрузке и, как следствие, для правильного распознавания стадии сердечной недостаточности. Пациенты, проходящие за 6 мин более 551 м не имеют признаков сердечной недостаточности; проходящие расстояние от 426 до 550 м относятся к I ФК, проходящие расстояние от 301 до 425 м - ко ІІ ФК, от 151 до 300 м - к III ФК, а пациенты, проходящие за 6 мин менее 150 м относятся к IV ФК. В последнее время к этой простой схеме классификации все чаще обращаются российские врачи. Значимость симптомов и клинических признаков чрезвычайно велика, поскольку именно они заставляют врача подозревать наличие у больного $\mathrm{CH}$.

\section{Клинические проявления сердечной недостаточности}

Критерии, используемые для диагностики ХCH, включают одышку разной тяжести, быструю утомляемость, сердцебиение, кашель и ортопноэ. У больных также могут быть периферические отеки, застойные хрипы в легких, тахикардия, набухшие яремные вены, гепатомегалия, ритм галопа и кардиомегалия. Необходимо убедиться, что эти симптомы связаны с заболеванием сердца, а не других систем и органов. Симптомы сердечной недостаточности могут усиливаться в связи с такими факторами, как транзиторная ишемия миокарда, тахикардия и брадиаритмии, тромбоэмболия легочной артерии, увеличение степени митральной регургитации, дисфункция почек, патология щитовидной железы, побочные эфффекты лекарственных средств, чрезмерное потребление поваренной соли и воды. Важными фракторами обострения симптомов являются также острая респираторная инфекция (до 25\% случаев декомпенсации ХCH связано с простудными заболеваниями) и злоупотребление алкоголем. По данным W.R. Harlan et al. (1977), F.V. Aguirre (1989) и S. Chakko (1992), из клинических симптомов наибольшее диагностическое значение имеют одышка (чувствительность $66 \%)$ и кардиомегалия (62\%), а также рентгенологические признаки застоя в легких (50\%).

Одышка возникает как результат усиленной работы системы дыхания и является наиболее частым симптомом сердечной недостаточности. На ранних стадиях сердечной недостаточности одышка возникает во время физической нагрузки, но по мере прогрессирования она появляется и при менее напряженной активности и даже сохраняется в покое. Активация рецепторов легких приводит к частому дыханию, и работа дыхательных мышц усиливается, а снабжение их кислородом уменьшается. Одной из причин появления одышки в положении лежа (ортопноэ) является перераспределение крови, что сопровождается повышением гидростатического давления. Больные с ортопноэ спят, приподняв верхнюю часть тела, и просыпаются от чувства нехватки воздуха. Это чувство обычно исчезает после того, как больной некоторое время посидит в вертикальном положении. По мере прогрессирования сердечной недостаточности ортопноэ настолько усиливается, что больной вынужден ночь проводить сидя в вертикальном положении. Угнетение дыхательного центра во время сна может сопровождаться снижением напряжение кислорода в артериальной крови. Это характерно для больных с интерстициальным отеком легких [34, 35].

Тяжелой формой сердечной астмы является острый отек легких, развивающийся вследствие повышения давления в капиллярах. Он может приводить к альвеолярному отеку, который сопровождается резкой нехваткой воздуха при дыхании, влажными хрипами над всеми легкими и отхаркиванием кровянистой жидкости. Одним из вариантов нарушения дыхания может быть дыхание Чейна-Стокса, которое отражает снижение чувствительности дыхательного центра. Изменения газового состава артериальной крови стимулируют пораженный дыхательный центр, приводя к гипервентиляции, за которыми следует апноэ. Появлению такой формы нарушения дыхания способствует артериальная гипертензия и коронарная болезнь сердца, сочетающиеся с поражением сосудов головного мозга. Утомляемость и слабость являются 
неспецифическими признаками, но часто встречаются при сердечной недостаточности. Отсутствие аппетита и одышка, сочетающиеся с болями и чувством тяжести в животе, являются типичными жалобами этих больных, которые связаны с застоем крови в венозной системе печени и селезенки [36, 37].

При тяжелой сердечной недостаточности у пациентов, страдающих церебральным атеросклерозом, наблюдаются нарушение памяти, затруднение концентрации внимания, изменение психического состояния, появление головных болей и бессонница. При умеренной сердечной недостаточности больные испытывают недомогание в тех случаях, когда находятся в горизонтальном положении длительное время. При более тяжелой сердечной недостаточности появляется цианоз губ и ногтевых лож, синусовая тахикардия, набухание яремных вен.

При обследовании возможен альтернирующий пульс, который, как правило, наблюдается у больных с кардиомиопатиями, артериальной гипертензией или ишемической болезнью сердца. У больных с сердечной недостаточностью часто выявляют влажные крепитирующие хрипы в нижних отделах легких и притупление при перкуссии нижних отделов легких. Нередко встречаются и сердечные отеки, локализация которых зависит от положения тела, но чаше обнаруживаются на нижних конечностях, в частности на лодыжках. Гидроторакс и асцит возникают при застойной сердечной недостаточности и проникновение жидкости в плевральные полости. Асцит также развивается вследствие транссудации жидкости. Застойная гепатомегалия проявляется также расширением, напряжением и пульсацией печени. Она наблюдается у больных с асцитом, но возможна и при менее тяжелых формах сердечной недостаточности. При длительной гепатомегалии одновременно может развиться и спленомегалия. На поздних стадиях застойной сердечной недостаточности появляются признаки желтухи [38, 39].

В случае острого возникновения застойных явлений в печени желтуха может иметь выраженный характер. При тяжелой хронической сердечной недостаточности может наблюдаться значительная потеря веса и развитие сердечной кахексии. В результате сердечной недостаточности конечности холодеют, приобретают бледную окраску, диурез понижается, а удельная плотность мочи повышается, в ней появляется белок. Рентгенографические исследования выявляют увеличение камер сердца и обнаруживаются изменения сосудов легких. Кроме того, на рентгенографии легких можно выявить плевральный выпот [40].

\section{Выводы.}

$\mathrm{XCH}$ определяется как «патофизиологический синдром, при котором в результате того или иного заболевания сердечно-сосудистой системы происходит снижение насосной функции, что приводит к дисбалансу между гемодинамической потребностью организма и возможностями сердца».

Распространенность ХСН в Российской Федерации составляет 7\% случаев, а терминальной XCH III-IV ФК достигает 2,1\% случаев. Этиологическими фракторами чаще всего являются артериальная гипертония, ИБС и сахарный диабет. $63 \%$ больных обращаются для лечения в стационар, а $37 \%$ - в поликлинику. Средний возраст среди амбулаторных больных составляет 59,6 лет, а среди стационарных больных 68,6 лет. Доля XCH с нормальной фракцией выброса
(ФВ > 40\%) среди амбулаторных больных составляет более $80 \%$.

При рассмотрении патогенеза сердечной недостаточности следует отметить, что ХCH - это синдром, развивающийся в результате различных заболеваний сердечно-сосудистой системы. Принципиальная новизна современных представлений о патогенезе XCH связана с тем фрактом, что ключевыми событиями на пути развития и прогрессирования $\mathrm{XCH}$ являются: заболевание сердечно-сосудистой системы; снижение сердечного выброса; задержка натрия и избыточной жидкости в организме. В 2001 г. были разработаны и рекомендованы этапы (стадии) сердечно-сосудистого континуума. Прогноз больных ХCH по-прежнему остается одним из самых плохих.

По поводу классификации хронической сердечной недостаточности ведется немало споров. В настоящее время в нашей стране все чаще используется классификация сердечной недостаточности, предложенная Нью-Йоркской ассоциацией кардиологов (NYHA) 1994 г. Согласно этой классификации, больные с синдромом сердечной недостаточности разделены на четыре функциональных класса.

Критерии, используемые для диагностики $\mathrm{XCH}$, включают одышку разной тяжести, быструю утомляемость, сердцебиение, кашель и ортопноэ. У больных также могут быть периферические отеки, застойные хрипы в легких, тахикардия, набухшие яремные вены, гепатомегалия, ритм галопа и кардиомегалия. Важными факторами обострения симптомов являются острая респираторная инфекция и злоупотребление алкоголем. Из клинических симптомов наибольшее диагностическое значение имеют одышка и кардиомегалия. Клинический диагноз ХCH обычно основывается на тщательном анализе жалоб, данных анамнеза и результатах физикального обследования. Установление диагноза XCH на ранних стадиях заболевания только на основании анализа клинических симптомов представляется довольной трудной задачей, поскольку они часто обусловлены влиянием ряда дополнительных факторов, включая возраст, ожирение и недостаточную тренированность. Повышенную утомляемость и одышку относят к наиболее частым и ранним клиническим проявлениям $\mathrm{XCH}$. Не существует какого-либо одного определенного диагностического признака ХСН [29].

По данным исследования IMPROVEMENT (2000 г.), самые частые жалобы больных XСН - одышка и быстрая утомляемость (98,4 и 94,3\% соответственно). Третьим по частоте симптомом является сердцебиение $(80,4 \%)$, а такие классические симптомы застоя, как периферические отеки, кашель, хрипы в легких и ортопноэ, вместо ожидаемых высоких мест занимают в списке более скромные позиции - лишь с четвертой по седьмую (с 73 до 28\%) [13].

Традиционный подход к объяснению патофизиологических механизмов развития симптомов $\mathrm{XCH}$ включает следующие рассуждения. Во-первых, нарушение фрункции сердца приводит к нарушению ответной реакции на физическую нагрузку. В свою очередь, это обусловливает недостаточное кровоснабжение мышц. В ответ на недостаточную перфузию из мышечной ткани в мозг поступают сигналы, которые могут восприниматься в виде ощущения повышенной утомляемости. Возникновение одышки объясняют чрезмерным повышением давления наполнения Лж, которое необходимо для обеспечения достаточного 
минутного объема сердца при выполнении физической нагрузки. Такое повышение приводит к застою в легких, а в тяжелых случаях - к выходу жидкой части крови в альвеолы [30].

\section{ЛИТЕРАТУРА}

1. Терещенко, С.Н. Хроническая сердечная недостаточность у женщин / С.Н. Терещенко, И.В. Жиров // Журнал Сердечная недостаточность. — 2008. — № 6. — С.295-299.

2. Smith, W.M. Epidemiology of congestive heart failure/W.M. Smith I/ Am. J. Cardiol. - 1985. — Vol. 55. - P.3A-8A.

3. Gibson, T.C. The prevalence of congestive heart failure in two rural communities / T.C. Gibson, K.L. White, L.M. Klainer // J. Chronic. Dis. - 1966. - Vol. 19. - P.141-152.

4. The epidemiology of heart failure: Framingham Study / K.K. Ho, J.L. Pinsky, W.B. Kannel [et. al.] // J. Am. Coll. Cardiol. - 1993. - Vol. 22 (suppl. A). - P.6A-13A.

5. Беленков, Ю.Н. Медикаментозные пути улучшения прогноза больных хронической сердечной недостаточностью / Ю.Н. Беленков, В.Ю. Мареев, Ф.Т. Агеев. - М.: Инсайт, 1997. - C.7.

6. Беленков, Ю.Н. Распространенность хронической сердечной недостаточности в Европейской части Российской Федерации. Данные ЭПОХА-ХСН (часть 2) / Ю.Н. Беленков, И.В. Фомин, В.Ю. Мареев [и др.] // Журнал Сердечная недостаточность. - 2006. - № 7 (3). - С.3-7.

7. Национальные рекомендации ВНОК И ОССН по диагностике и лечению XСH (третий пересмотр).

8. Cleland, J.G. The EuroHeart Failure survey programe a survey on the quality of care among patients with heart failure in Europe. Part 1:atient characteristics and diagnosis / J.G. Cleland, K. Swedberg, F. Follath [et al.] // Eur. Heart. J. - 2003. - Vol. 24 (5). - P.442-463.

9. Мареев, В.Ю. От имени рабочей группы исследования ЭПОХА-О-ХСН. Сравнительная характеристика больных с ХСН в зависимости от величины ФВ по результатам Российского многоцентрового исследования ЭПОХАО-ХСН / В.Ю. Мареев, М.О. Даниелян, Ю.Н. Беленков // Журнал Сердечная недостаточность. - 2006 - № 7 (4). - C.164-171.

10. Fox, K.F. Coronary artery disease as the cause of incident heart failure in the population / K.F. Fox, M.R. Cowie, A.J.S. Coats [et al.] // Eur. Heart. J. — 2001. — Vol. 22 (3). P.228-236

11. Ho, K. Survival after the onset of congestive heart failure in Framingham heart study subjects / K. Ho, K.M. Anderson, W.B. Kannel [et al.] // Circulation. — 1993. — Vol. 88 (1). P. $107-115$.

12. Schocken, D.D. Prevalence and mortality rate of congestive heart failure in the United States / D.D. Schocken, M.I. Arrieta, P.E. Leaverton, E.A. Ross // J. Am. Coll. Cardiol. — 1992. Vol. 20 (2). - P.301-306.

13. Davies, M. Prevalence of left-ventricular systolic dysfunction and heart failure in the Echocardiographic Heart of England Screening study: a population based study / M. Davies, F. Hobbs, R. Davis [et al.] // Lancet. - 2001. - Vol. 358 (9280). - P.439-444.

14. Halm, M.A. Heart failure in women / M.A. Halm, S. Penque // Prog. Cardiovasc Nurs. - 2000. - Vol. 15 (4). - P.121133.

15. American Heart Association. Heart and Stroke Statistical Update. National Center, 7272 Greenville Avenue. — Dallas, Texas, 2002. - P.38.

16. Бадин, Ю.В. Выживаемость больных ХСН в когортной выборке Нижегородской области (данные 1998-2002 годов) / Ю.В. Бадин, И.В. Фомин // Сердечная недостаточность: Всерос. конф. ОССН, 2005 г. - М., 2005. - С.31-32.

17. Даниелян, М.О. Прогноз и лечение хронической сердечной недостаточности (данные 20-летнего наблюдения): автореф. дис. ... канд. мед. наук / М.О. Даниелян. - М., 2001.

18. Беленков, Ю.Н. Эпидемиология и прогноз хронической сердечной недостаточности / Ю.Н. Беленков, Ф.Т. Агеев // Русский медицинский журнал. — 1999. — № 2.
19. Беленков, Ю.Н. Медикаментозные пути улучшения прогноза больных хронической сердечной недостаточностью / Ю.Н. Беленков, В.Ю. Мареев, Ф.Т. Агеев. - М.: Инсайт, 1997. - $80 \mathrm{c}$

20. Sutton, G.C. Epidemiologic aspects of heart failure / G.C. Sutton // Am. Heart. J. — 1990. — Vol. 120 (6, pt. 2). P.1538-1540.

21. McKeep, $A$. The natural history of congestive heart failure: the Framingham study / A. McKeep, W. Castlli, P. McNamara // N. Engl. J. Med. —1971. — Vol. 285 (26). - P.1441-1446.

22. Ho, K.K. The epidemiology of heart failure: the Framingham study / K.K. Ho, J.L. Pinsky, W.B. Kannel [et al.] // J. Am. Coll. Cardiol. - 1993. - Vol. 22 (4, suppl. A). - P.6A-13A

23. Фомин, И.В. Эпидемиология хронической сердечной недостаточности в Российской Федерации / И.В. Фомин // Хроническая сердечная недостаточность. - М.: ГЭОТАРМедиа, 2010. - С.7-77.

24. Щербинина, Е.В. Динамика этиологических причин фрормирования ХCH в репрезентативной выборке Нижегородской области за 9 лет наблюдения (1998-2007) / Е.В. Щербинина, Ю.В. Бадин, А.Р. Вайсберг [и др.] // Сердечная недостаточность: Всерос. конф. ОССН, 2007. - М., 2007. - С.38.

25. Беленков, Ю.Н. Определение понятия ХCH, причины развития, патогенез, классификация и цели терапии / Ю.Н. Беленков, В.Ю. Мареев. // Консилиум медикум.

26. Бойцов, С.А. Центральные и периферические механизмы / С.А. Бойцов // Журнал Сердечная недостаточность. 2005. - № 2. - C.1-5.

27. Pugnatores, S.A. Pars media et periphericis mechanisms of defectum cordis / S.A. Pugnatores // Journal. - 2005. № 2. - C.1.

28. Бенца, Т.М. К вопросу о лечении хронической сердечной недостаточности у больных ишемической болезнью сердца / Т.М. Бенца.

29. Dzau, V. Resolved and unresolved issues in the prevention and treatment of coronary artery disease: a workshop consensus statement / V. Dzau, E. Braunwald // Am. Heart. J. - 1991. - Vol. 121 (4 pt). - P.1244-1263.

30. Беленков, Ю.Н. Вклад различных факторов риска в сердечно-сосудистую смертность. Что их объединяет? Пленум / Ю.Н. Беленков // Сердце. - 2006. № 5(5). -С.1-3. - Приложение к журналу.

31. Gillum, R.F. Pulse rate, coronary heart disease and death: NHANES I epidemiologic follow-up study / R.F. Gillum, D.M. Makuc, J.J. Feldman [et al.] // Am. Heart J. — 1991. — Vol. 121. - P.172-177.

32. Терещенко, С.Н. Систолическая функция левого желудочка в развитии хронической сердечной недостаточности и ингибиторы АПФ / С.Н. Терещенко // Consilium Medicum. 2002. - Т. 4, № 11.

33. Национальные рекомендации ВНОК и ОССН по диагностике и лечению ХСН (третий пересмотр) (Утверждены конференцией ОССН 15 октября 2009 г.) // Журнал Сердечная недостаточность. - 2010. — № 1 (57). — С.3.

34. Окороков, А.Н. Диагностика болезней внутренних органов. Миокардиты, сердечная недостаточность / А.Н. Окороков. - М.: Мед. литература, 2004. - Т. 8. - 378 с.

35. Калюжин, В.В. Хроническая сердечная недостаточность / В.В. Калюжин, О.В. Калюжин, А.Т. Тепляков, А.В. Караулов. - М.: Мед. информ. агентство, 2005. - 288 с.

36. Кушаковский, М.С. Хроническая застойная сердечная недостаточность. Идиопатические миокардиопатии / М.С. Кушаковский. - СПб.: Фолиант, 1997. - 320 с.

37. Воронков, Л.Г. Хроническая сердечная недостаточность в схемах и таблицах / Л.Г. Воронков. - Киев: Инфо-Ф, 2002. $-134 \mathrm{c}$.

38. Бурдули, Н.М. Хроническая сердечная недостаточность / Н.М. Бурдули. - М.: Феникс, 2007. - 176 с.

39. Гуревич, М.А. Хроническая сердечная недостаточность / М.А. Гуревич. - М.: Практическая медицина, 2008. - 418 с.

40. Руководство по амбулаторно-поликлинической кардиологии / под ред. Ю. Беленкова, Р. Оганова. - М.: ГЭОТАРМедиа, 2007. - 400 с. 


\section{REFERENCES}

1. Tereschenko, S.N. Hronicheskaya serdechnaya nedostatochnost' u zhenschin / S.N. Tereschenko, I.V. ZHirov // Zhurnal Serdechnaya nedostatochnost'. — 2008. - № 6. S.295-299.

2. Smith, W.M. Epidemiology of congestive heart failure / W.M. Smith // Am. J. Cardiol. — 1985. — Vol. 55. - P.3A8A.

3. Gibson, T.C. The prevalence of congestive heart failure in two rural communities / T.C. Gibson, K.L. White, L.M. Klainer // J. Chronic. Dis. - 1966. - Vol. 19. - P.141-152.

4. The epidemiology of heart failure: Framingham Study K.K. Ho, J.L. Pinsky, W.B. Kannel [et. al.] // J. Am. Coll. Cardiol. - 1993. - Vol. 22 (suppl. A). - P.6A-13A.

5. Belenkov, Yu.N. Medikamentoznye puti uluchsheniya prognoza bol'nyh hronicheskoi serdechnoi nedostatochnost'yu / Yu.N. Belenkov, V.Yu. Mareev, F.T. Ageev. - M.: Insait, 1997. - S.7.

6. Belenkov, Yu.N. Rasprostranennost' hronicheskoi serdechnoi nedostatochnosti v Evropeiskoi chasti Rossiiskoi Federacii. Dannye EPOHA-HSN (chast' 2) / Yu.N. Belenkov, I.V. Fomin, V.Yu. Mareev [i dr.] // Zhurnal Serdechnaya Nedostatochnost'. — 2006. - № 7 (3). - S.3-7.

7. Nacional'nye rekomendacii VNOK I OSSN po diagnostike i lecheniyu HSN (tretii peresmotr).

8. Cleland, J.G. The EuroHeart Failure survey programe a survey on the quality of care among patients with heart failure in Europe. Part 1:atient characteristics and diagnosis / J.G. Cleland, K. Swedberg, F. Follath [et al.] // Eur. Heart. J. - 2003. - Vol. 24 (5). - P.442-463.

9. Mareev, V.Yu. Ot imeni rabochei gruppy issledovaniya EPOHA-O-HSN. Sravnitel'naya harakteristika bol'nyh s HSN v zavisimosti ot velichiny FV po rezul'tatam Rossiiskogo mnogocentrovogo issledovaniya EPOHA-O-HSN / V.Yu. Mareev, M.O. Danielyan, Yu.N. Belenkov // Zhurnal Serdechnaya nedostatochnost'. - 2006 - № 7 (4). S.164-171.

10. Fox, K.F. Coronary artery disease as the cause of incident heart failure in the population / K.F. Fox, M.R. Cowie, A.J.S. Coats [et al.] // Eur. Heart. J. — 2001. — Vol. 22 (3). P.228-236.

11. Ho, K. Survival after the onset of congestive heart failure in Framingham heart study subjects / K. Ho, K.M. Anderson, W.B. Kannel [et al.] // Circulation. — 1993. — Vol. 88 (1). P. $107-115$

12. Schocken, D.D. Prevalence and mortality rate of congestive heart failure in the United States / D.D. Schocken, M.I. Arrieta, P.E. Leaverton, E.A. Ross // J. Am. Coll. Cardiol. - 1992. Vol. 20 (2). - P.301-306.

13. Davies, M. Prevalence of left-ventricular systolic dysfunction and heart failure in the Echocardiographic Heart of England Screening study: a population based study / M. Davies, F. Hobbs, R. Davis [et al.] // Lancet. - 2001. - Vol. 358 (9280). - P.439-444.

14. Halm, M.A. Heart failure in women / M.A. Halm, S. Penque // Prog. Cardiovasc Nurs. - 2000. - Vol. 15 (4). - P.121133.

15. American Heart Association. Heart and Stroke Statistical Update. National Center, 7272 Greenville Avenue. — Dallas, Texas, 2002. - P.38

16. Badin, Yu.V. Vyzhivaemost' bol'nyh HSN v kogortnoi vyborke Nizhegorodskoi oblasti (dannye 1998-2002 godov) / Yu.V. Badin, I.V. Fomin // Serdechnaya nedostatochnost': Vseros. konf. OSSN, 2005 g. - M., 2005. - S.31-32.

17. Danielyan, M.O. Prognoz i lechenie hronicheskoi serdechnoi nedostatochnosti (dannye 20-letnego nablyudeniya): avtoref. dis. ... kand. med. nauk / M.O. Danielyan. - M., 2001.

18. Belenkov, Yu.N. Epidemiologiya i prognoz hronicheskoi serdechnoi nedostatochnosti / Yu.N. Belenkov, F.T. Ageev // Russkii medicinskii zhurnal. — 1999. — № 2

19. Belenkov, Yu.N. Medikamentoznye puti uluchsheniya prognoza bol'nyh hronicheskoi serdechnoi nedostatochnost'yu
/ Yu.N. Belenkov, V.Yu. Mareev, F.T. Ageev. - M.: Insait, 1997. - $80 \mathrm{~s}$.

20. Sutton, G.C. Epidemiologic aspects of heart failure / G.C. Sutton // Am. Heart. J. - 1990. - Vol. 120 (6, pt. 2). P.1538-1540

21. McKeep, A. The natural history of congestive heart failure: the Framingham study / A. McKeep, W. Castlli, P. McNamara // N. Engl. J. Med. -1971. — Vol. 285 (26). — P.14411446.

22. Ho, K.K. The epidemiology of heart failure: the Framingham study / K.K. Ho, J.L. Pinsky, W.B. Kannel [et al.] // J. Am. Coll. Cardiol. - 1993. - Vol. 22 (4, suppl. A). - P.6A-13A.

23. Fomin, I.V. Epidemiologiya hronicheskoi serdechnoi nedostatochnosti $\vee$ Rossiiskoi Federacii / I.V. Fomin // Hronicheskaya serdechnaya nedostatochnost'. - M.: GEOTAR-Media, 2010. - S.7-77.

24. Scherbinina, E.V. Dinamika etiologicheskih prichin formirovaniya HSN v reprezentativnoi vyborke Nizhegorodskoi oblasti za 9 let nablyudeniya (1998-2007) / E.V. Scherbinina, Yu.V. Badin, A.R. Vaisberg [i dr.] // Cerdechnaya nedostatochnost': Vseros. konf. OSSN, 2007. - M., 2007. - S.38.

25. Belenkov, Yu.N. Opredelenie ponyatiya HSN, prichiny razvitiya, patogenez, klassifikaciya i celi terapii / Yu.N. Belenkov, V.Yu. Mareev. // Konsilium medikum.

26. Boicov, S.A. Central'nye i perifericheskie mehanizmy / S.A. Boicov // Zhurnal Serdechnaya Nedostatochnost'. 2005. - № 2. - S.1-5.

27. Pugnatores, S.A. Pars media et periphericis mechanisms of defectum cordis / S.A. Pugnatores // Journal. - 2005. № 2. - C.1.

28. Benca, T.M. K voprosu o lechenii hronicheskoi serdechnoi nedostatochnosti u bol'nyh ishemicheskoi bolezn'yu serdca / T.M. Benca.

29. Dzau, V. Resolved and unresolved issues in the prevention and treatment of coronary artery disease: a workshop consensus statement / V. Dzau, E. Braunwald // Am. Heart. J. - 1991. - Vol. 121 (4 pt). - P.1244-1263.

30. Belenkov, Yu.N. Vklad razlichnyh faktorov riska v serdechnososudistuyu smertnost'. CHto ih ob'edinyaet? Plenum / Yu.N. Belenkov // Serdce. — 2006. — № 5 (5). — S.1-3. Prilozhenie k zhurnalu.

31. Gillum, R.F. Pulse rate, coronary heart disease and death: NHANES I epidemiologic follow-up study / R.F. Gillum, D.M. Makuc, J.J. Feldman [et al.] // Am. Heart J. — 1991. — Vol. 121. - P.172-177.

32. Tereschenko, S.N. Sistolicheskaya funkciya levogo zheludochka $\checkmark$ razvitii hronicheskoi serdechnoi nedostatochnosti i ingibitory APF / S.N. Tereschenko // Consilium Medicum. - 2002. T. 4, № 11 .

33. Nacional'nye rekomendacii VNOK i OSSN po diagnostike lecheniyu HSN (tretii peresmotr) (Utverzhdeny konferenciei OSSN 15 oktyabrya 2009 g.) // Zhurnal Serdechnaya nedostatochnost'. - 2010. - № 1 (57). - S.3.

34. Okorokov, A.N. Diagnostika boleznei vnutrennih organov. Miokardity, serdechnaya nedostatochnost' / A.N. Okorokov. M.: Med. literatura, 2004. - T. 8. - $378 \mathrm{~s}$.

35. Kalyuzhin, V.V. Hronicheskaya serdechnaya nedostatochnost'/ V.V. Kalyuzhin, O.V. Kalyuzhin, A.T. Teplyakov, A.V. Karaulov. M.: Med. inform. agentstvo, 2005. - $288 \mathrm{~s}$.

36. Kushakovskii, M.S. Hronicheskaya zastoinaya serdechnaya nedostatochnost'. Idiopaticheskie miokardiopatii / M.S. Kushakovskii. — SPb.: Foliant, 1997. - 320 s.

37. Voronkov, L.G. Hronicheskaya serdechnaya nedostatochnost' v shemah i tablicah / L.G. Voronkov. — Kiev: Info-F, 2002. $134 \mathrm{~s}$.

38. Burduli, N.M. Hronicheskaya serdechnaya nedostatochnost' / N.M. Burduli. - M.: Feniks, 2007. - 176 s.

39. Gurevich, M.A. Hronicheskaya serdechnaya nedostatochnost' / M.A. Gurevich. - M.: Prakticheskaya medicina, 2008. $418 \mathrm{~s}$.

40. Rukovodstvo po ambulatorno-poliklinicheskoi kardiologii / pod red. Yu. Belenkova, R. Oganova. - M.: GEOTAR-Media, 2007. $-400 \mathrm{~s}$. 